\title{
Immuno-oncology and its history
}

Keywords: immuno-oncology, malignancy, BRM, streptococcus pyogenes, CML, NHL

\section{Introduction}

Immuno-oncology is a branch of medicine which employs a human's inherent immune system to combat malignancy and cancer. It is one of the most promising branch of medical science in a world where every year around 14.1 million people are diagnosed with cancer. Also referred to as biological response modifier (BRM) therapy, biologic therapy, biotherapy, cancer immunotherapy or IO therapy, it involves either modification of the patient's own immune system to strengthen it and increase its capacity to fight the disease, or, injecting man-made proteins which imitate and assist the patient's own immune cells to stop/prevent cancer proliferation and malignancy. While some therapies work on an 'overall' basis, some are very specific. ${ }^{1}$

The inception of IO therapies can be traced back to more than $100 y e a r s$, when the surgical oncologist William Coley (also known as the Father of Immunotherapy) induced a hypothesis, stating that stirring the patient's immune system by introducing a host of live and attenuated bacteria could compel various forms of tumors to attain complete remission. (He introduced strains of Streptococcus pyogenes and Serratia marcescens into patients with malignancies like sarcoma, lymphoma and testicular carcinoma achieving positive results like durable complete remission). However, a lack of knowledge about the exact mechanism and potency of the bacteria, a risk of purposely promoting inflammation among already affected patients, along with the simultaneous introduction of radiotherapy and surgical procedures led to the doctors opting for the latter. ${ }^{1-3}$

Since then, oncology research has constantly stressed upon the importance of immunotherapy in cancer treatment. The first approval for an immune-oncologic therapy for cancer came in 1986 in the form of Interferon Alpha, a class of immune regulators, to be administered in patients with Hairy Cell leukemia, chronic myelogenous leukemia (CML), follicular non-Hodgkin lymphoma (NHL), melanoma, and AIDS-related Kaposi's sarcoma. This was gradually followed by a number of approvals including the Tuberculosis vaccine (BCG: Bacillus Calmette-Guérin), InterLeukin-2 (IL-2), Trastuzumab, Rituximab, Gardasil and so on. ${ }^{4}$

In due course of years of research, scientists and researchers have derived upon the conclusion that the safest mode of treatment of cancer is elicitation of an immune response within the patient's system, which screens and eliminates cancerous and tumorous cells, with few or no side-effects. Normally, the innate (in-born) branch of immunity provides the first line of defense against possible tumor antigens, be it viral proteins, mutated or abnormally expressed cellular proteins which have the potential to cause cancerous lesions. It does so by recognizing the antigenic proteins with the help of the MHC (major histocompatibility complex) and the dendritic cells, which process these viral proteins and present it to the cytotoxic T cells (CD4 and CD8) for complete eradication of these antigens. The adaptive immune system acts as a 'memory' unit with the B-cells producing targeted antibodies to eradicate similar entities in future - a process known as immunosurveillance. Some cells however, have the capacity
Volume 6 Issue I - 2018

Sreetama Dutt

Scientific Content Writer, The University of Manchester, UK

Correspondence: Sreetama Dutt, Scientific Content Writer,WEbRx Lifesystems Pvt. Ltd., Bangalore, India,Te +919874700697, Email sreetama.dutt@gmail.com

Received: September 09, 2017 | Published: January 05, 2018

to escape this immunosurveillance mechanism and reverse the same in their favor, allowing the tumor to spread and lead to malignancy and metastasis - a phenomenon known as tumor immune tolerance. ${ }^{5}$

To combat this acquired immune tolerance among cancer patients, the immune system needs to be stimulated, which is where immunotherapy comes into play. Immunotherapy employs different methods to activate different components of the immune system, by either infusing immune cells or antibodies which trigger appropriate immune responses. Application of these therapies has been shown to benefit patients on a long-term basis, with their effects lasting in patients for months and even years after termination of the therapy. This combined with the fact that immunotherapy involves a selective selection of only cancerous cells, the other cells are spared from the cytotoxicity involved in conventional therapies like radiation and chemotherapy -makes immunotherapy the best possible option for treating cancer patients. ${ }^{4,6}$

It is to be remembered that conventional, standard-of-care cancer therapies (like radiation and chemotherapy) do possess some vital immunomodulatory functions like making some of the tumor cells more recognizable to the immune system which are otherwise masked by mutations and aberrations, eradicating cells which cause immune suppression (like regulatory $\mathrm{T}$ cells and granulocytic myeloid-derived suppressor cells). So a combination regimen with both conventional as well as immunotherapeutic techniques might lead to increased specificity and selectivity, hence amount to increased tolerability and safety, when applied in cancer patients. In a nutshell, it can be said that, instead of conventional regimens that target the immune system on a whole, the targeted immunotherapies target specifically the tumors at specific stages to modulate specific immune responses in favor of the patient. ${ }^{2,4,6}$

\section{Acknowledgements}

None.

\section{Conflict of interest}

The author declares no conflict of interest.

\section{References}

1. American Cancer Society. Evolution of Cancer Treatments: Immunotherapy. 2017.

2. http://www.10forio.info/understanding-immuno-oncology\#is-immunetherapy-a-cure-for-cancer 
3. William BC. A Brief History of Immunotherapy. Targeted Oncology 2014.

4. www.immunooncologynow.com

5. Dunn GP, Old LJ, Schreiber RD. The Immunobiology of Cancer Immunosurveillance and Immunoediting. Immunity. 2004;21(2):137148
6. Parish CR. Cancer immunotherapy: The past, the present and the future. Immunol Cell Biol. 2003;81(2):106-113. 